\title{
Polydopamine-Assisted Fabrication of Stable Silver Nanoparticles on Optical Fiber for Enhanced Plasmonic Sensing
}

\author{
Yiwen TANG ${ }^{1 \dagger}$, Hui YUAN ${ }^{1 \dagger}$, Jiangping $\mathrm{CHEN}^{1 \dagger}$, Qiguo XING ${ }^{1}$, \\ Rongxin $\mathrm{SU}^{1,2 *}$, Wei $\mathrm{QI}^{1,2}$, and Zhimin $\mathrm{HE}^{1}$
}

\begin{abstract}
${ }^{1}$ State Key Laboratory of Chemical Engineering, Tianjin Key Laboratory of Membrane Science and Desalination Technology, School of Chemical Engineering and Technology, Tianjin University, Tianjin 300072, China

${ }^{2}$ Collaborative Innovation Center of Chemical Science and Engineering (Tianjin), Tianjin 300072, China
\end{abstract}

$\dagger$ These authors contributed equally to this work

*Corresponding author: Rongxin SUＥ-mail: surx@tju.edu.cn

\begin{abstract}
We present a facile and effective method for fabrication of the localized surface plasmon resonance (LSPR) optical fiber sensor assisted by two polydopamine (PDA) layers with enhanced plasmonic sensing performance. The first PDA layer was self-polymerized onto the bare optical fiber to provide the catechol groups for the reduction from $\mathrm{Ag}^{+}$to $\mathrm{Ag}^{\circ}$ through chelating and redox activity. As the reduction of $\mathrm{Ag}^{+}$proceeds, $\mathrm{Ag}$ nanoparticles (NPs) were grown in-situ on the PDA layer with uniform distribution. The second PDA layer was applied to prevent Ag NPs from oxidating and achieve an improvement of LSPR signal. The PDA/Ag/PDA-based optical fiber sensor has an enhanced LSPR sensitivity of $961 \mathrm{~nm} / \mathrm{RIU}$ and excellent oxidation resistance. The stable PDA/Ag/PDA-based LSPR sensor with high optical performance is very promising for future application in optical sensing field.
\end{abstract}

Keywords: LSPR; optical fiber; polydopamine; in-situ growth; silver nanoparticles

Citation: Yiwen TANG, Hui YUAN, Jiangping CHEN, Qiguo XING, Rongxin SU, Wei QI, and Zhimin HE, "Polydopamine-Assisted Fabrication of Stable Silver Nanoparticles on Optical Fiber for Enhanced Plasmonic Sensing," Photonic Sensors, 2020, 10(2): 97-104.

\section{Introduction}

Localized surface plasmon resonance (LSPR), a phenomenon occurs when electromagnetic field of oscillating electrons on the surface of nanoparticles (NPs) matches that of incident light, creates prominent spectral absorption and scattering peaks along with strong electromagnetic near-field enhancement. LSPR is normally associated with noble metal nanostructures and has been widely applied in drug screening, biological detection, and disease diagnosis [1-4], due to its high sensitivity of localized electromagnetic field around the metal surfaces upon the change in environmental refractive index (RI).

Up to date, there are four approaches to fabricate LSPR sensors, i.e., electron-beam lithography, physical evaporation, electrostatic self-assembly (ESSA), and in-situ synthesis. Duyne et al. reported the use of nanosphere lithography for building silver nanospheres with regular arrangement [5-7]. They

Received: 29 January 2019 / Revised: 5 May 2019

(C) The Author(s) 2019. This article is published with open access at Springerlink.com DOI: $10.1007 / \mathrm{s} 13320-019-0564-7$

Article type: Regular 
utilized the self-assembly of size-monodisperse polystyrene nanospheres as the uniform deposition mask. With thermal or electron beam evaporation, metal was deposited through the mask. Then, the metal NPs remained on the substrates in order after removing the polystyrene nanospheres. Jia et al. fabricated $\mathrm{Au} / \mathrm{Ag}$ bimetallic NPs by evaporating silver onto gold modified glass through a transmission electron microscopy (TEM) copper grid to build well-ordered patterns [8]. ESSA is another method to build LSPR sensors by achieving the interactions between negatively charged NPs and positively charged glass substrates. The experimental conditions including $\mathrm{pH}$, temperature, solution concentration, and fabrication times together with the size and shape of NPs could contribute to the differences of sensitivity, the limit of detection, and the resolution of optical fiber sensors [9-17].

Dopamine (DA) is an organic chemical of phenethylamine and catecholamine families that can aggregate and form a polydopamine (PDA) layer and then interact with almost all types of substrates [18]. Since the mechanism of PDA formation is still not well known, some evidences have suggested two possible routes including noncovalent self-assembly and covalent polymerization [19]. It was generally accepted that the covalent polymerization plays a key role in the initial stages of deposition. As the aggregation continues, noncovalent interactions become more dominant [20]. Usually, a short time is sufficient for the deposition of the PDA layer in a stirred solution, and the deposition promotes a morphologically uniform and continuous PDA film for further surface-mediated reactions [21].

Considering the strong adhesion and quick deposition capability of PDA [22], along with the high plasmonic activities of silver nanostructures on account of their appropriate dielectric functions and electronic structures [2, 23], we developed an effective strategy to prepare $\mathrm{PDA} / \mathrm{Ag} / \mathrm{PDA}$-based
LSPR optical fiber sensor. In our work, the first PDA layer serves as reductant for the reduction from free $\mathrm{Ag}^{+}$in solution to $\mathrm{Ag}$ NPs on substrate. Then the second PDA layer was deposited on the Ag NPs-modified surface to prevent oxidation and increase the sensitivity of the sensor.

\section{Experimental section}

\subsection{Materials}

Multimode optical fibers with a 0.22-numerical aperture and a 400- $\mu \mathrm{m}$ core diameter were purchased from Ocean Optics Inc. Dopamine hydrochloride was purchased from Sigma-Aldrich. Silver nitrate $\left(\mathrm{AgNO}_{3}\right)$ and tris (hydroxymethyl) aminomethane were purchased from Aladdin Reagent Co., Ltd. (China). All of the other chemicals used were of analytical grade.

\subsection{Experimental setup}

The bifurcated optical fiber (SPLIT-400-VISNIR, Ocean Optics) served as a connection to the LSPR sensor on one of its terminals and a tungsten-halogen light source (DH-2000-BAL, Ocean Optics) along with a mini-spectrometer (USB2000, Ocean Optics) on the bifurcated side. The other terminal of the sensor, which has been fabricated with sensing layer, was dipped into a certain solution. The device was operated by the following way, i.e., a white light emits from the light source to excite the LSPR effect of the sensor and the spectrometer assists in capturing the optical signal reflected by the silver layer of the sensor. Finally, a computer linked with the spectrometer was used to monitor the optical signal.

\subsection{Fabrication of the LSPR sensor}

Figure 1 demonstrates the fabrication process of the PDA/Ag/PDA-based LSPR optical sensor. Firstly, the bare optical fiber was immersed in piranha solution (7:3 mixture of concentrated $\mathrm{H}_{2} \mathrm{SO}_{4}$ and $30 \% \mathrm{H}_{2} \mathrm{O}_{2}$ ) for $30 \mathrm{~min}$ at $90{ }^{\circ} \mathrm{C}$ and then it was rinsed with deionized (DI) water and dried in $\mathrm{N}_{2}$ 
stream. After that, the piranha-cleaned optical fiber was functionalized by DA through a polymerization reaction, during which optical fiber was immersed in freshly prepared DA solution $(1 \mathrm{mg} / \mathrm{mL}$ in $10 \mathrm{mM}$ Tris buffer, $\mathrm{pH}$ 8.5) under stirring with a speed of $500 \mathrm{rpm}$ for $10 \mathrm{~min}$. Then this optical fiber was immediately rinsed again with DI water and dried with $\mathrm{N}_{2}$ stream. Secondly, the PDA-fabricated optical fiber was immersed in $\mathrm{AgNO}_{3}$ solution
$(10 \mathrm{mM})$ for $18 \mathrm{~h}$. In this step, $\mathrm{Ag}^{+}$was reduced to $\mathrm{Ag}^{\mathrm{O}}$ by catechol groups in PDA and the color of optical fiber surface changed from transparent to yellowish. Thirdly, the Ag NPs-based optical fiber was modified by PDA again using the same procedure as that of the first PDA layer. At last, a thick layer of $\mathrm{Ag}(\sim 200 \mathrm{~nm})$ was coated on the end of sensing area through silver mirror reaction to promote reflecting the light to the spectrometer.

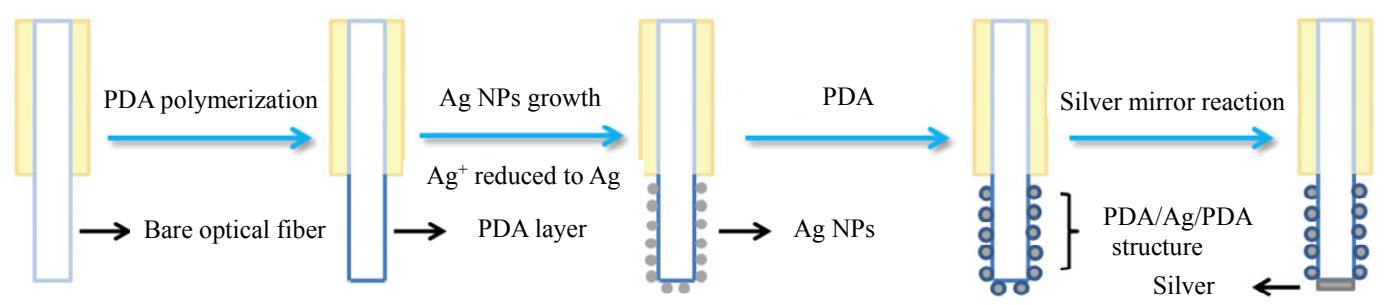

Fig. 1 Schematic of the fabrication process of PDA/Ag/PDA-based LSPR sensor in this work.

\subsection{Numerical Method}

The electric field distributions of the Ag NPs were calculated by the 3D finite-difference time-domain (FDTD) method [24], and their geometric parameters are determined based on the scanning electron microscopy (SEM) images of the fabricated sensors. All the dielectric functions of $\mathrm{Ag}$ and glass were obtained from Palik database [25]. Override mesh region with $0.8 \mathrm{~nm}$ mesh size is used to enclose the whole NP to get accurate results. To verify the accuracy and stability of the calculations, convergence testing was done before all the simulations. All the calculations of electric field distributions for the Ag NPs were done at their corresponding resonant wavelengths. In this study, all the calculations were performed using the FDTD simulation program (FDTD solutions 8.6, Lumerical solutions, Inc., Vancouver, Canada).

\section{Results and discussion}

\subsection{In-situ synthesis of Ag NPs on PDA-modified optical fiber surface}

Since DA can self-polymerize to PDA on almost all the material surfaces in weakly alkaline and aerobic conditions [15], we utilized a PDA layer to modify a bare optical fiber. In the $\mathrm{AgNO}_{3}$ solution, the catechol groups in PDA reduced the $\mathrm{Ag}^{+}$to $\mathrm{Ag}^{\mathrm{o}}$. As a result, Ag NPs were formed on optical fiber from the aggregated neighboring $\mathrm{Ag}^{\mathrm{O}}$ clusters, leading to the fabrication of PDA/Ag-based sensor. The SEM image in Fig. 2(a) shows a number of separate Ag NPs on the sensor surface and help identify the morphology of the silver film. The Ag NPs appeared to distribute in a relatively uniform size. Moreover, there was no apparent aggregation of Ag NPs on optical fiber. Figure 2(b) shows the statistical graph of the Ag NPs distribution on the LSPR sensor surface. With the help of the Nano Measure (a software), we obtained the statistics of particle distribution (200 points picked at random). According to the statistical result, the NPs distributed in an almost uniform size between $20 \mathrm{~nm}-30 \mathrm{~nm}$, which can contribute to the excellent signal of the final sensor.

To study the sensing ability of the fabricated PDA/Ag-based LSPR sensor, we measured the reflectivity spectra of the sensor in various solvents 


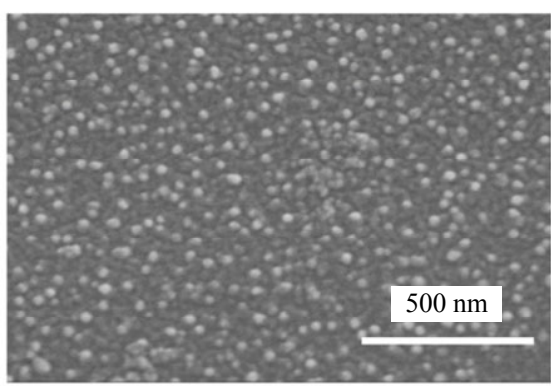

(a)

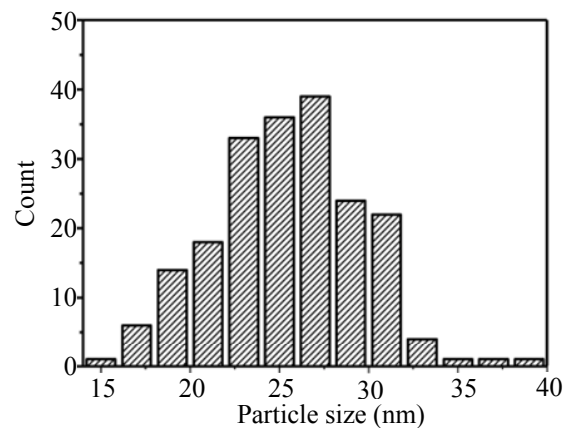

(b)

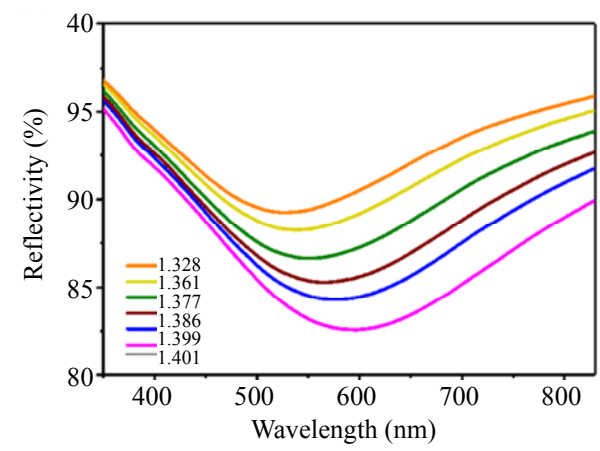

(c)

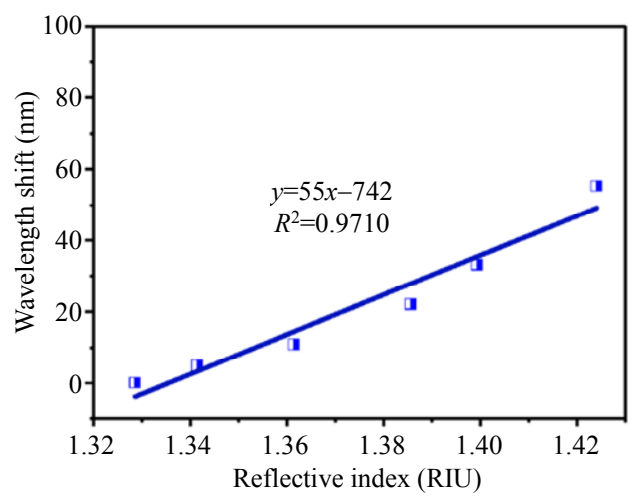

(d)

Fig. 2 Characterizations of the as-prepared sensor: (a) SEM images of $\mathrm{Ag} \mathrm{NPs}$ coated on optical fiber, (b) histogram showing the corresponding particle size distribution of Ag NPs, (c) the reflective spectra of the PDA/Ag-based LSPR sensor in different alcoholic solution, and (d) changes in the LSPR wavelength shift of the as-prepared sensor to the RI. with RI ranging from 1.328 to 1.401 . Table 1 shows the detailed solvents and corresponding RI. As shown in Figs. 2(c) and 2(d), the resonant wavelength redshifted with an increase in the RI, and a linear relationship is obtained between the resonant wavelength shift and the solvent RI. The refractive index sensitivity (RIS) was calculated by $S_{n}=\delta \lambda_{\text {res }} / \delta n_{s}$, where $\delta n_{s}$ is the refractive index change of the solution, and $\delta \lambda_{\text {es }}$ is the corresponding resonance wavelength shift. As shown in Fig. 2(d), the sensor shows a sensitivity up to $555 \mathrm{~nm} /$ RIU. This sensitivity was higher than that of the Ag NPs prepared by photodeposition, physical lithography, and electrostatic self-assembly [26-29], as shown in Table 1.

To confirm the high sensitivity of the as-prepared Ag NPs-based optical sensor, we calculated the electric field distributions of the Ag NPs on an optical fiber under resonance conditions by using the FDTD method. The diameter and height were set to $25 \mathrm{~nm}$ and $12.5 \mathrm{~nm}$, respectively, according to the mean geometric values measured in the experiments. As shown in Fig. 3(a), strongly enhanced electric fields were generated close to the Ag NPs surface, especially in the space near the marked optical fiber. The LSPR peak of the simulated Ag NP arrays was at $502 \mathrm{~nm}$ in water, which was in agreement with the measured spectrum in experiment. Moreover, the SEM image in Fig. 2(a) shows that some Ag NPs were close to each other, which might generate interparticle coupling. Hence, we further calculated the electric field distributions of the dimers with gap sizes of $0 \mathrm{~nm}, 10 \mathrm{~nm}$, and 15 nm. As shown in Figs. 3(b)-3(d), interparticle junction in the dimers generated greater enhancement of electric fields, which indicated strong interparticle coupling. For dimers with $0 \mathrm{~nm}$, $10 \mathrm{~nm}$, and $15 \mathrm{~nm}$ gaps, the greatest electric field enhancements at the gap area were $4 \times 10^{3}, 2.8 \times 10^{3}$, $2.8 \times 10^{3}$, respectively, all of which were larger than that of the single Ag NPs with the value of $1.4 \times 10^{3}$. 
Table 1 Sensitivity comparisons of the fabricated PDA/Ag/PDA-based LSPR sensor with other Ag sensors.

\begin{tabular}{|c|c|c|c|c|}
\hline Samples & Methods & Applications & LSPR sensitivity (nm/RIU) & Reference \\
\hline Ag NPs & Photodeposition & LSPR & $\begin{array}{c}387 \\
(R I=1.333-1.442)\end{array}$ & [26] \\
\hline Ag NPs & Photodeposition & LSPR & $\begin{array}{c}68 \\
(R I=1.0-1.5)\end{array}$ & [27] \\
\hline Ag nano-disks & Lithography & LSPR & $\begin{array}{c}325 \\
(R I=1.333-1.378)\end{array}$ & [28] \\
\hline Ag NPs & Electrostatic self-assembly & LSPR & $\begin{array}{c}387 \\
(R I=1.33-1.40)\end{array}$ & [29] \\
\hline PDA/Ag-based & In-situ reduction & LSPR & $\begin{array}{c}555 \\
(R I=1.33-1.40)\end{array}$ & This work \\
\hline PDA/Ag/PDA-based & $\begin{array}{l}\text { In-situ reduction+ } \\
\text { PDA modification }\end{array}$ & LSPR & $\begin{array}{c}961 \\
(R I=1.33-1.40)\end{array}$ & This work \\
\hline
\end{tabular}
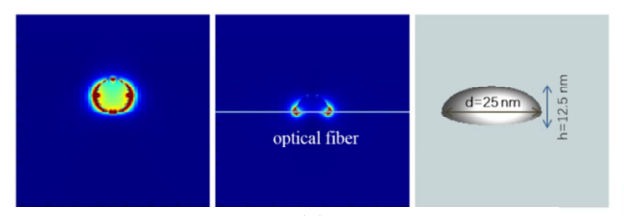

(a)
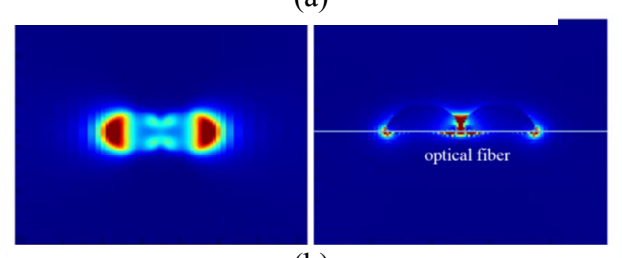

(b)
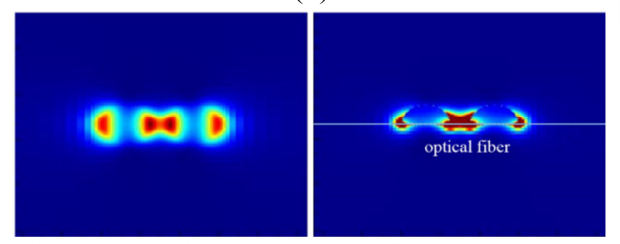

(c)
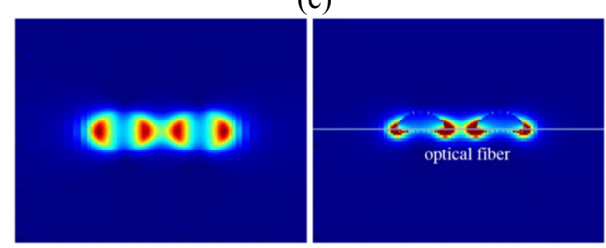

(d)

Fig. 3 Calculated electric field distributions $\left(|E|^{2} /\left|E_{0}\right|^{2}\right)$ of the Ag NPs on the optic fiber surface when the particles existed as (a) a single NPs, (b) a dimer without gap, (c) a dimer with a $10 \mathrm{~nm}$ gap, and (d) a dimer with a $15 \mathrm{~nm}$ gap (the left demonstrates the top view; the right shows the side view).

Therefore, the good sensing performance of the PDA/Ag-based sensor might be partly ascribed to the interparticle coupling of the dimers. Based on the simulation results, constructed Ag NPs arrays present LSPR peaks at $562 \mathrm{~nm}, 548 \mathrm{~nm}$, and $534 \mathrm{~nm}$ for the regions of dimers with $0 \mathrm{~nm}, 10 \mathrm{~nm}$, and $15 \mathrm{~nm}$ gaps in water, respectively, which is in good agreement with the experiment.

To further confirm the coupling effect of the dimers, we simulated the spectra of single AgNPs and dimers on optical fiber in different alcoholic solutions. The simulation of single AgNPs and dimers in solutions were carried out while RI ranging from 1.328 to 1.401 . The resonance wavelength shifts $38 \mathrm{~nm}$ from $502 \mathrm{~nm}$ to $540 \mathrm{~nm}$ for single NPs and the dimers without gap shows $49 \mathrm{~nm}$ shift from $562 \mathrm{~nm}$ to $611 \mathrm{~nm}$ in the same environmental RI range. Moreover, as shown in Fig. 2(b), the sensitivity of the sensor fabricated in experiment is $555 \mathrm{~nm} / \mathrm{RIU}$, which is between $511 \mathrm{~nm} / \mathrm{RIU}$ and $574 \mathrm{~nm} / \mathrm{RIU}$. As a result, the simulated work confirmed that dimers may exist on the PDA/Ag-based optical fiber sensor, which can contribute to the improvement of sensitivity.

\subsection{LSPR performance and stability of PDA/Ag/PDA optical fiber sensor}

To further protect $\mathrm{Ag}$ NPs and increase the sensitivity of PDA/Ag-based optical fiber sensor, the second PDA layer was modified on the fiber surface.

We immersed the sensing area of our sensor in various solvents with RI ranging from 1.328 to 1.401 to examine the sensitivity of $\mathrm{PDA} / \mathrm{Ag} / \mathrm{PDA}$ - 


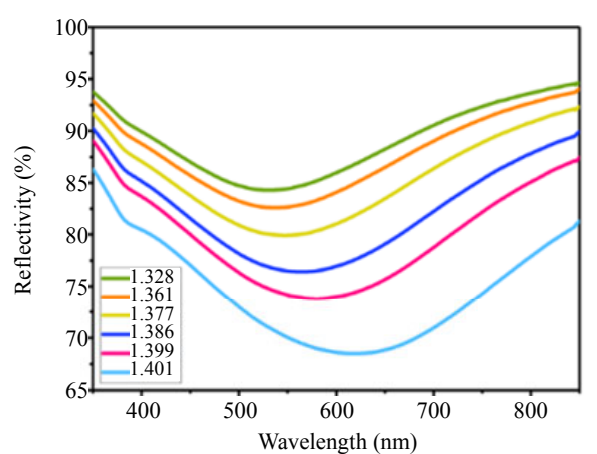

(a)

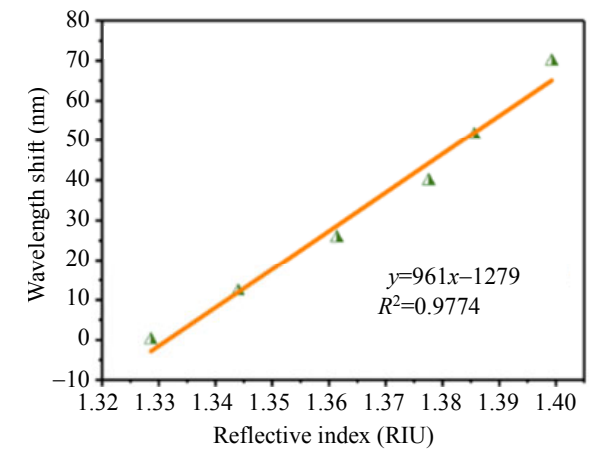

(b)

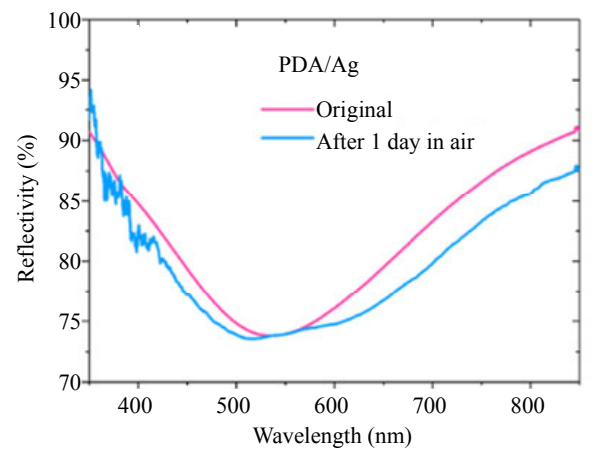

(c)

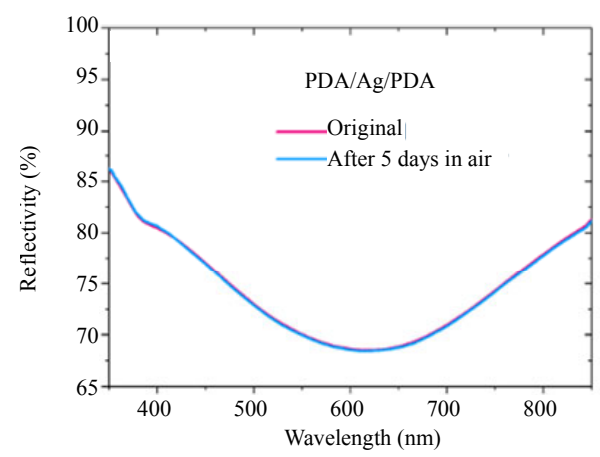

(d)

Fig. 4 LSPR performance and stability of PDA/Ag/PDA optical fiber sensor: (a) the reflective spectra of the PDA/Ag/PDA-based LSPR sensors in different alcoholic solutions, (b) changes in the LSPR wavelength shift of the as-prepared sensors to the RI, (c) reflectivity spectra of the PDA/Ag-based LSPR sensor, (c) PDA/Ag/PDA-based LSPR sensor and (d) in butanol before treatment and after storage in the air. based LSPR sensor. As shown in Fig. 4(a), reflectivity peaks illustrate that $\mathrm{PDA} / \mathrm{Ag} / \mathrm{PDA}$ sensor has stronger LSPR signal. In the RI range of 1.328-1.401, the sensor shows brilliant optical performance with the enhanced sensitivity of 961 $\mathrm{nm} / \mathrm{RIU}$. As shown in Table 1, the Ag NPs prepared by photodeposition, physical lithography, and electrostatic self-assembly show lower sensitivity than two constructed sensors in this work [26-29]. Moreover, the sandwich-structured PDA/Ag/PDAbased sensor presents higher sensitivity compared with the PDA/Ag-based sensor. The improved sensitivity is most likely due to the redshift of the resonance wavelength. It has been reported that the redshift of spectrum could enhance the sensitivity of SPR sensors [30]. Therefore, the additional layer of PDA, which contributed to the redshift of the spectra, played an important role in increasing the sensitivity of the optical fiber sensor. As shown in Fig. 4(c), the FWHM of the reflectivity spectra of the PDA/Ag-based LSPR sensor after 1 day in air increased because of the deformation of the Ag NPs resulting from their poor chemical and structural stabilities. In comparison, as shown in Fig.4(d), the reflectivity spectra of the PDA/Ag/PDA-based LSPR sensor after 5 days in air almost overlapped with the original spectrum. The results proved the excellent oxidation resistance of the PDA/Ag/PDA, indicating that the second PDA layer on Ag NPs prevented them from oxidating. Due to its enhanced sensitivity and stability, PDA/Ag/PDA-based LSPR sensor is very promising in the future application.

\section{Conclusions}

In summary, we reported a facile method for the fabrication of PDA/Ag/PDA-based LSPR sensor in this work. The method is based on the strong self-polymerization capability of PDA and the in-situ growth of Ag NPs for LSPR sensing. The first PDA layer worked as a reductant and a binder to modify the Ag NPs to the optical fiber surface, whereas the second PDA layer was coated again to 
prevent oxidation and increase the sensitivity of the sensor. The prepared PDA/Ag/PDA-based LSPR sensor presented improved LSPR performance and stability owing to the key role of PDA on the fabrication process. Compared with the PDA/Ag-based and other Ag NPs-based LSPR sensors, the PDA/Ag/PDA-based LSPR sensor had an excellent sensitivity of $961 \mathrm{~nm} / \mathrm{RIU}$. The sandwich-structured sensor showed an obvious enhancement in sensitivity and stability, which demonstrated that PDA could be of great value to the performance enhancement of optical fiber sensors.

\section{Acknowledgment}

This work was supported by the National Natural Science Foundation of China (Grant No. 51473115), Tianjin Municipal Science and Technology Bureau, China (Grant Nos. 18YFZCSF00590 and 18YFHBZC00010), and Wuqing S\&T Commission (Grant Nos. WQKJ201726 and WQKJ201806).

Open Access This article is distributed under the terms of the Creative Commons Attribution 4.0 International License (http://creativecommons.org/licenses/by/4.0/), which permits unrestricted use, distribution, and reproduction in any medium, provided you give appropriate credit to the original author(s) and the source, provide a link to the Creative Commons license, and indicate if changes were made.

\section{References}

[1] E. C. Peláez, M. C. Estevez, A. Portela, J. P. Salvador, M. P. Marco, and L. M. Lechuga, "Nanoplasmonic biosensor device for the monitoring of acenocoumarol therapeutic drug in plasma," Biosensors and Bioelectronics, 2018, 119: $149-155$.

[2] Y. Wang, S. Meng, Y. Liang, L. Li, and W. Peng, "Fiber-optic surface plasmon resonance sensor with multi-alternating metal layers for biological measurement," Photonic Sensors, 2013, 3(3): 202-207.

[3] M. T. Alula, L. Karamchand, N. R. Hendricks, and J. M. Blackburn, "Citrate-capped silver nanoparticles as a probe for sensitive and selective colorimetric and spectrophotometric sensing of creatinine in human urine," Analytica Chimica Acta, 2018, 1007: 40-49.

[4] Q. Jiang, M. Xue, P. Liang, C. Zhang, J. Lin, and J. Ouyang, "Principle and experiment of protein detection based on optical fiber sensing," Photonic Sensors, 2017, 7(4): 317-324.

[5] M. D. Malinsky, K. L. Kelly, G. C. Schatz, and R. P. Van Duyne, "Chain length dependence and sensing capabilities of the localized surface plasmon resonance of silver nanoparticles chemically modified with alkanethiol self-assembled monolayers," Journal of the American Chemical Society, 2001, 123(7): 1471-1482.

[6] C. L. Haynes, A. J. Haes, A. D. McFarland, and R. P. Van Duyne, "Nanoparticles with tunable localized surface plasmon resonances," Radiative Decay Engineering, Springer, Boston, MA, 2005, pp. 47-99.

[7] J. Zhao, X. Zhang, C. R. Yonzon, A. J. Haes, and R. P. Van Duyne, "Localized surface plasmon resonance biosensors," Nanomedicine, 2006, 1(2): 219-228.

[8] K. Jia, M. Y. Khaywah, Y. Li, J. L. Bijeon, P. M. Adam, R. Déturche, et al., "Strong improvements of localized surface plasmon resonance sensitivity by using $\mathrm{Au} / \mathrm{Ag}$ bimetallic nanostructures modified with polydopamine films," ACS Applied Materials \& Interfaces, 2014, 6(1): 219-227.

[9] M. Consales, M. Pisco, and A. Cusano, "Lab-on-fiber technology: a new avenue for optical nanosensors," Photonic Sensors, 2012, 2(4): 289-314.

[10] B. Sciacca and T. M. Monro, "Dip biosensor based on localized surface plasmon resonance at the tip of an optical fiber," Langmuir, 2014, 30(3): 946-954.

[11] T. J. Lin and M. F. Chung, "Detection of cadmium by a fiber-optic biosensor based on localized surface plasmon resonance," Biosensors and Bioelectronics, 2009, 24(5): 1213-1218.

[12] Y. Q. Chen and C. J. Lu, "Surface modification on silver nanoparticles for enhancing vapor selectivity of localized surface plasmon resonance sensors," Sensors and Actuators B: Chemical, 2009, 135(2): 492-498.

[13] K. J. Chen and C. J. Lu, “A vapor sensor array using multiple localized surface plasmon resonance bands in a single UV-vis spectrum," Talanta, 2010, 81(4): 1670-1675.

[14] J. Cao, M. H. Tu, T. Sun, and K. T. V. Grattan, "Wavelength-based localized surface plasmon resonance optical fiber biosensor," Sensors and Actuators B: Chemical, 2013, 181: 611-619.

[15] Y. Shao, S. Xu, X. Zheng, Y. Wang, and W. Xu, "Optical fiber LSPR biosensor prepared by gold nanoparticle assembly on polyelectrolyte 
multilayer," Sensors, 2010, 10(4): 3585-3596.

[16] S. Shi, L. Wang, R. Su, B. Liu, R. Huang, W. Qi, and Z. He, "A polydopamine-modified optical fiber SPR biosensor using electroless-plated gold films for immunoassays," Biosensors \& Bioelectronics, 2015, 74: 454-460.

[17] Y. Chen and H. Ming, "Review of surface plasmon resonance and localized surface plasmon resonance sensor," Photonic Sensors, 2012, 2(1): 37-49.

[18] H. Lee, S. M. Dellatore, W. M. Miller, and P. B. Messersmith, "Mussel-inspired surface chemistry for multifunctional coatings," Science, 2007, 318(5849): 426-430.

[19] S. Hong, Y. S. Na, S. Choi, I. T. Song, W. Y. Kim, and H. Lee, "Non-covalent self-assembly and covalent polymerization co-contribute to polydopamine formation," Advanced Functional Materials, 2012, 22(22): 4711-4717.

[20] N. F. D. Vecchia, R. Avolio, M. Alfè, M. E. Errico, A. Napolitano, and M. D'Ischia, "Building-block diversity in polydopamine underpins a multifunctional eumelanin-type platform tunable through a quinone control point," Advanced Functional Materials, 2013, 23(10): 1331-1340.

[21] R. A. Zangmeister, T. A. Morris, and M. J. Tarlov, "Characterization of polydopamine thin films deposited at short times by autoxidation of dopamine," Langmuir, 2013, 29(27): 8619-8628.

[22] S. Shi, L. B. Wang, A. K. Wang, R. L. Huang, L. Ding, R. X. Su, et al., "Bioinspired fabrication of optical fiber SPR sensors for immunoassays using polydopamine-accelerated electroless plating," Journal of Materials Chemistry C, 2016, 4(32): 7554-7562.

[23] C. Gao, Y. Hu, M. Wang, M. Chi, and Y. Yin,
"Fully alloyed Ag/Au nanospheres: combining the plasmonic property of ag with the stability of Au," Journal of the American Chemical Society, 2014, 136(20): 7474-7479.

[24] A. Taflove and S. C. Hagness, Computational electrodynamics: the finite-difference time-domain method. Boston, United States: Artech House, 2005.

[25] E. D. Palik and G. Ghosh, Handbook of optical constants of solids. San Diego, United States: Academic Press, 1998.

[26] T. Liu, W. Wang, F. Liu, and S. Wang, "Photochemical deposition fabricated highly sensitive localized surface plasmon resonance based optical fiber sensor," Optics Communications, 2018, 427: 301-305.

[27] J. G. Ortega-Mendoza, A. Padilla-Vivanco, C. Toxqui-Quitl, N. P. Zaca-Morã, N. D. Villegas-Hernã, and V. F. Chã, "Optical fiber sensor based on localized surface plasmon resonance using silver nanoparticles photodeposited on the optical fiber end," Sensors, 2014, 14(10): 18701-18710.

[28] N. A. Cinel, B. Serkan, and Z. Ekmel, "Electron beam lithography designed silver nano-disks used as label free nano-biosensors based on localized surface plasmon resonance," Optics Express, 2012, 20(3): 2587.

[29] J. P. Chen, S. Shi, R. X. Su, W. Qi, R. L. Huang, M. F. Wang, et al., "Optimization and application of reflective lspr optical fiber biosensors based on silver nanoparticles," Sensors, 2015, 15(6): 12205-12217.

[30] O. Tabasi and C. Falamaki, "Recent advancements in the methodologies applied for the sensitivity enhancement of surface plasmon resonance sensors," Analytical Methods, 2018, 10(32): 3906-3925. 